\title{
Screening and preliminary validation of T lymphocyte immunoregulation-associated long non-coding RNAs in diabetic foot ulcers
}

\author{
SHUJUAN XU ${ }^{1}$, XIAOYU WENG ${ }^{1}$, YA WANG $^{1}$, DALUN LV $^{2}$, MEIHONG ZENG ${ }^{1}$, FEN ZHAO $^{1}$ and YU SUN ${ }^{1}$ \\ Departments of ${ }^{1}$ Surgery and ${ }^{2}$ Burn and Plastic Surgery, Yijishan Hospital, \\ The First Affiliated Hospital of Wannan Medical College, Wuhu, Anhui 241001, P.R. China
}

Received June 13, 2018; Accepted December 6, 2018

DOI: $10.3892 / \mathrm{mmr} .2019 .9877$

\begin{abstract}
The present study aimed to investigate the existence of immunoregulation-associated long non-coding (lnc)RNAs mediated by $\mathrm{T}$ lymphocytes in the wound surfaces of diabetic foot ulcers (DFUs). The wound skin tissues of patients receiving debridement for trauma or DFUs associated with infection were obtained. Dermatological histological changes were observed by pathological staining, and T lymphocyte subsets and inflammation-associated cytokines were identified. Gene chip technology was used to screen for lncRNAs regulated by immune cells. The wound skin structure in the control group was revealed to be complete, and the inflammatory response was not marked. However, the wound skin structure in the ulcer group was disordered and exhibited a notable inflammatory response. Compared with the control group, expression levels of cluster of differentiation (CD) 3 and CD8 in the wound surface tissues of the ulcer group were significantly increased, while the expression levels of interleukin (IL)-1 $\beta$, IL-2, IL-10, interferon- $\gamma$ and tumor necrosis factor- $\alpha$ were significantly upregulated. A target regulatory association was identified between downregulated lncRNA-ENST00000411554 and upregulated mitogen-activated protein kinase (MAPK) 1 in DFU tissues, and a negative correlation was detected between this RNA and protein. The present results suggested that an immune functional disorder of $\mathrm{T}$ lymphocytes may be closely associated with the development of DFUs. Furthermore, activation of the MAPK signal transduction pathway mediated by the lncRNA-ENST00000411554/MAPK1 axis may affect the DFU immune regulatory imbalance.
\end{abstract}

Correspondence to: Mrs. Shujuan Xu, Department of Surgery, Yijishan Hospital, The First Affiliated Hospital of Wannan Medical College, 2 Zheshan West Road, Wuhu, Anhui 241001, P.R. China E-mail: 485227129@qq.com

Key words: diabetic foot ulcers, T lymphocytes, long non-coding RNA, mitogen-activated protein kinase signaling pathway

\section{Introduction}

Recently, the incidence of diabetic mellitus (DM) has sharply increased; it has become one of the major diseases affecting quality of life and represents a threat to public health (1). The World Health Organization has predicted that the number of patients with DM will increase to 366 million worldwide by 2030 (2). An epidemiological study in China in 2010 reported that the incidence of DM in adults was $11.6 \%$, while the overall incidence of DM in the population was $50.1 \%$ (3).

Diabetic foot ulcers (DFUs) commonly occur in patients with DM, and represent one of the principal chronic and severe complications associated with the disease. DFUs may arise from peripheral neuropathy, lower limb vasculopathy-associated foot infections and ulcerations. The most serious outcomes of DFUs are amputation or mortality; thus, DFUs may severely affect the quality of life of patients with DM and their families $(4,5)$. As the incidence of DM increases, the number of patients with DFUs is also increasing, with $\sim 15 \%$ of patients with DM worldwide suffering from DFUs, and this number is even higher in China (25\%) (6). The resulting series of clinical and social problems caused by DFUs has attracted scientific interest, and comprehensive investigation of DFU pathogenesis and refractory mechanisms has become an important research topic.

The immunopathological effect mediated by T lymphocytes has been reported to have an important role in the occurrence and development of DM $(7,8)$. Typically, genetic and immune factors can promote damage to islet $\mathrm{B}$ cells, while metabolic disorders, environmental factors and the secretion of glucocorticoids may inhibit the function of lymphocytes (9). Long-term hyperglycemia and the glycosylation of cell membranes may also damage the function of granulocytes and attenuate the response of $\mathrm{T}$ cells to mitosis (10). However, despite numerous studies having investigated immune dysfunction in patients with type $2 \mathrm{DM}$, the underlying molecular mechanisms remain unclear.

Human genome transcription products with no protein coding activity have been demonstrated to exhibit biological functions, rather than merely existing as transcriptional noise. For example, long non-coding (lnc)RNAs are RNA molecules with lengths of $>200$ nucleotides that do not exhibit protein 
coding activity; however, IncRNAs do have genetic regulatory functions. Numerous studies have demonstrated that abnormal lncRNA expression is closely associated with DM and its complications. Sequencing of the human $\beta$ cell transcriptome identified 1,128 pancreas islet-specific lncRNAs, a number of which were associated with pancreatic differentiation (11). Gao et al (12) revealed that the expression of H19 lncRNA is significantly decreased in the skeletal muscle of patients with type $2 \mathrm{DM}$ and in animals with insulin resistance, and suppressed expression of $\mathrm{H} 19$ results in interference of the insulin signal in myocytes and reduced glucose uptake, which suggests that H19 is involved in the glycometabolism of DM. Furthermore, a study investigating diabetic retinopathy demonstrated that lncRNA-p21 inhibits the proliferation of vascular smooth muscle cells and macrophages, and induces apoptosis via regulation of cellular tumor antigen p53 signaling (13). In addition, rs2648875, rs13447075 and rs2648862 lncRNAs are closely associated with the occurrence and development of diabetic nephropathy (14).

Despite numerous studies having investigated lncRNAs, DM and its complications, few studies have investigated the immunoregulation of IncRNAs in DFUs. Therefore, in the present study, the $\mathrm{T}$ lymphocyte subset and differences in the expression levels of inflammation-associated cytokines in DFU tissues were investigated. In addition, lncRNAs exhibiting target regulatory associations with immune cells and relevant factors were screened for using gene chip technology to determine the immunoregulation and potential molecular mechanism of lncRNAs associated with the occurrence and development of DFUs. The results of the present study may identify molecular targets for the prevention and treatment of DFUs.

\section{Materials and methods}

Tissue specimen collection. A total of 39 patients with DFU and 22 trauma patients receiving debridement at Yijishan Hospital, Wannan Medical College (Wuhu, China) between January and February 2017 were enrolled. A total of 3 patients with DFU and 3 trauma patients were randomly divided into an ulcer group and a control group. The ulcer group contained one man and two women aged 50-69 years, and the control group contained one man and two women aged 48-65 years. There were no significant differences between the two groups. Patients with DM were excluded from the control group, which included patients whose wounds were caused by trauma, and for whom debridement indications were clear. Patients with a confirmed diagnosis of type 2 DM were included in the ulcer group. Their lower limb ulcers had persisted for longer than 1 month with no healing following medical treatment. Debridement indications were again clear for this group.

Complete skin tissues $(2 \times 2 \mathrm{~cm})$ were collected from the center of the wound surfaces from patients in the two groups. Following washing, a number of tissue samples were fixed in $4 \%$ paraformaldehyde for $48 \mathrm{~h}$ at $25^{\circ} \mathrm{C}$ and embedded in paraffin. The remaining tissues were stored at $-80^{\circ} \mathrm{C}$. The present study was approved by the Ethics Committee of Yijishan Hospital, and all patients provided written informed consent.
Pathological staining. Wound surface tissues were fixed with $4 \%$ paraformaldehyde, washed in purified water, dehydrated in ethanol, cleared by purified water, immersed in paraffin, embedded and sectioned at $5 \mu \mathrm{m}$. The sections were further dewaxed at $25^{\circ} \mathrm{C}$, hydrated at $25^{\circ} \mathrm{C}$ and stained using hematoxylin and eosin (HE) for $30 \mathrm{sec}$ at $25^{\circ} \mathrm{C}$ and Masson's trichome for $40 \mathrm{sec}$ at $25^{\circ} \mathrm{C}$ (Gibco; Thermo Fisher Scientific, Inc., Waltham, MA, USA) to observe histological changes, including alterations associated with blood capillaries, collagen and fibroblasts.

Immunofluorescent staining of tissue specimens. Skin tissues were frozen for $30 \mathrm{~min}$ at $-20^{\circ} \mathrm{C}$ and cut into sections $(8 \mu \mathrm{m})$. The sections were fixed in paraformaldehyde for 1 day at $25^{\circ} \mathrm{C}$, washed a number of times with distilled water and subsequently dried at room temperature. Following this, the sections were treated with pure acetone at $4^{\circ} \mathrm{C}$ for $10 \mathrm{~min}$ and dried prior to further immunostaining. The sections were washed with PBS and $0.3 \%$ Triton X-100 for 30 min at $37^{\circ} \mathrm{C}$, washed again with PBS and blocked using $10 \%$ fetal bovine serum (Gibco; Thermo Fisher Scientific, Inc.) for $30 \mathrm{~min}$ at $37^{\circ} \mathrm{C}$. Primary antibodies against forkhead box P3 (1:100; Abcam, Cambridge, UK; cat. no. ab4728), cluster of differentiation (CD)3 (1:200; Abcam; cat. no. ab5690), CD4 (1:200; Abcam; cat. no. ab203034) or CD8 (1:150; Abcam; cat. no. ab4055) were added and incubated with the sections at $4^{\circ} \mathrm{C}$ overnight. Following washing with PBS, membranes were incubated at $37^{\circ} \mathrm{C}$ for $30 \mathrm{~min}$ with an appropriate fluorescent secondary antibody (1:500; Invitrogen; Thermo Fisher Scientific, Inc.; cat. no. A-11034). Finally, sections were stained with DAPI for $5 \mathrm{~min}$ at $37^{\circ} \mathrm{C}$, washed with PBS and observed via laser confocal scanning microscopy at x100 magnification (Leica Microsystems GmbH, Wetzlar, Germany).

Western blot analysis. The total protein was extracted from the skin tissue using radioimmunoprecipitation assay buffer (Invitrogen; Thermo Fisher Scientific, Inc.), and its concentration was measured using a bicinchoninic acid assay kit (Invitrogen; Thermo Fisher Scientific, Inc.). The remaining protein was degenerated by boiling at $99^{\circ} \mathrm{C}$ for $10 \mathrm{~min}$, and was subsequently stored at $-20^{\circ} \mathrm{C}$. Protein samples $(20 \mu \mathrm{g})$ were run on $10 \%$ SDS-PAGE gels and transferred onto polyvinylidene fluoride membranes for $80 \mathrm{~min}$. Membranes were blocked with $5 \%$ bovine serum albumin (Invitrogen; Thermo Fisher Scientific, Inc.) at $25^{\circ} \mathrm{C}$ for $1 \mathrm{~h}$ and incubated at $4^{\circ} \mathrm{C}$ overnight with primary antibodies (all purchased from Abcam) against the following proteins: Transforming growth factor- $\beta$ (TGF- $\beta$; $1: 5,000$; cat. no. ab92486), interleukin (IL)-1 $\beta$ (1:1,000; cat. no. ab2105), IL-2 (1:1,000; cat. no. ab180780), IL-6 (1:500; cat. no. ab6672), IL-10 (1:1,000; cat. no. ab34843), IL-8 (1:1,000; cat. no. ab7747), interferon (IFN)- $\gamma(1: 400$; cat. no. ab25101), tumor necrosis factor (TNF)- $\alpha$ (1:2,000; cat. no. ab6671) and GAPDH (1:5,000; cat. no. ab8245), $\beta$-actin (1:5,000; cat. no. ab8226). Following this, the membranes were washed three times with TBS containing $0.1 \%$ Tween-20 (TBST; $10 \mathrm{~min} /$ wash) and incubated with a secondary antibody (1:5,000; Cell Signaling Technology, Inc., Danvers, MA, USA) at room temperature for $1 \mathrm{~h}$. Membranes were subsequently washed three times with TBST (10 min/wash) and developed 
using a gel imaging system (EMD Millipore, Billerica, MA, USA).

Microarray analysis of the IncRNA expression profile. There were at least three replicates in the control test. Therefore, three tissue specimens were randomly selected from each group to conduct the IncRNA gene chip assay. The other tissue specimens from the patients were used for further test and verify. RNA was extracted from three randomly selected skin tissue samples obtained from the control and ulcer groups using TRIzol ${ }^{\circledR}$ reagent (Invitrogen; Thermo Fisher Scientific, Inc.). Quantified RNA was transcribed into fluorescent-labeled complementary (c)RNA using the Quick Amp Labeling kit (Agilent Technologies, Inc., Santa Clara, CA, USA) at $65^{\circ} \mathrm{C}$. Following this, cRNAs were hybridized onto the Arraystar Human LncRNA Microarray v4.0 (Arraystar Inc., Rockville, $\mathrm{MD}, \mathrm{USA}$ ) and the fluorescence intensity was determined using an Agilent G2565BA microarray scanner (Agilent Technologies, Inc.). Images were input into Agilent Feature Extraction software version 10.5.1, and the data underwent quantile normalization and further analysis using Agilent Gene Spring software GX 12.0 (both Agilent Technologies, Inc.). A total of 20 lncRNAs and co-expressing mRNAs exhibiting maximum levels of upregulation and downregulation were screened for and verified via reverse transcription quantitative polymerase chain reaction (RT-qPCR), using GAPDH as an internal reference.

$R T-q P C R$. Total RNA was extracted from control and ulcer tissues using TRIzol ${ }^{\circledR}$, according to the manufacturer's instructions. IncRNA and mRNA expression levels were determined using the Ribo ${ }^{\mathrm{TM}}$ SYBR Green mRNA/lncRNA RT-qPCR Starter kit (Guangzhou RiboBio Co., Ltd., Guangzhou, China), using GAPDH as an internal reference. Realtime PCR system (2XMaster Mix: $5 \mu 1,10 \mu \mathrm{M}$ PCR-specific primer F: $0.5 \mu 1$, $10 \mu \mathrm{M}$ PCR-specific primer R: $0.5 \mu 1$ water added for total volume: $8 \mu \mathrm{l}$ ) was used to prepare all the DNA samples for PCR reaction. The conditions for RT-qPCR were: $95^{\circ} \mathrm{C}$ for $10 \mathrm{~min} ; 40$ cycles, then $95^{\circ} \mathrm{C}$ for $10 \mathrm{sec}$ and $60^{\circ} \mathrm{C}$ for $60 \mathrm{sec}$. Dissociation curves revealed no nonspecific amplification. The primers used for the RT-qPCR assay are listed in Tables I and II. The results were analyzed using the $2^{-\Delta \Delta C q}$ method (15), and all experiments were repeated in triplicate.

IncRNA fluorescence in situ hybridization (FISH). An lncRNA antisense probe was synthesized by Novatech Enterprise Co., Ltd. (Wuxi, China). Skin tissues were embedded in paraffin, cut into sections $(4 \mu \mathrm{m})$, dewaxed and sealed with sealing solution for $15 \mathrm{~min}$. Pre-hybridization solution (100-150 $\mu \mathrm{l}$, Novatech Enterprise Co., Ltd. Wuxi, China) was added to the sections, which were incubated at $37^{\circ} \mathrm{C}$ for $1 \mathrm{~h}$. The lncRNA antisense probe was diluted in pre-hybridization solution, degenerated at $65^{\circ} \mathrm{C}$ for $5 \mathrm{~min}$ and rapidly cooled in iced water for $10 \mathrm{~min}$. Following this, the pre-hybridization solution was discarded from the sections, which were uniformly covered with 100-150 $\mu \mathrm{l}$ degenerated probe and incubated at $4^{\circ} \mathrm{C}$ overnight. The sections were subsequently washed, sealed for 30 min with sealing solution at $37^{\circ} \mathrm{C}$ and then stained with DAPI for 1-5 min at room temperature. Following this, the sections were washed with running water, air-dried, sealed
Table I. Primer sequences for reverse transcription-quantitative polymerase chain reaction.

\begin{tabular}{|c|c|}
\hline Gene & Primer sequence $\left(5^{\prime}-3^{\prime}\right)$ \\
\hline \multirow[t]{2}{*}{ TGF- $\beta$} & F: CGCGTGCTAATGGTGGAAA \\
\hline & R: CGCTTCTCGGAGCTCTGATG \\
\hline \multirow[t]{2}{*}{ TNF- $\alpha$} & F: GCCAGAGGGCTGATTAGAGA \\
\hline & R: TCAGCCTCTTCTCCTTCCTG \\
\hline \multirow[t]{2}{*}{$\mathrm{IFN}-\gamma$} & F: GCTCTAGAGATTTCAACTTCTTTGGCTTA \\
\hline & R:TTGTCGACGCAGGCAGGACAACCATTACT \\
\hline \multirow[t]{2}{*}{ IL-1 $\beta$} & F: CCGACCACCACTACAGCAAG \\
\hline & R: TGGACCAGACATCACCAAGC \\
\hline \multirow[t]{2}{*}{ IL-2 } & F: ATGTACAGGATGCAACTCCTG \\
\hline & $\begin{array}{l}\text { R: TCAAGTCAGTGTTGAGATGATGCTTTG } \\
\text { ACAAAA }\end{array}$ \\
\hline \multirow[t]{2}{*}{ IL-6 } & F: ATGAACTCCTTCTCCACAAGC \\
\hline & $\begin{array}{l}\text { R: CTACATTTGCCGAAGAGCCCTCAGGCTG } \\
\text { GACTG }\end{array}$ \\
\hline \multirow[t]{2}{*}{ IL-8 } & F: ATGACTTCCAAGCTGGCCGTG \\
\hline & $\begin{array}{l}\text { R: TTATGAATTCTCAGCCCTCTTCAAAA } \\
\text { ACTTCTC }\end{array}$ \\
\hline \multirow[t]{2}{*}{ IL-10 } & F: AACCTGCCTAACATGCTTCG \\
\hline & R: GCAAGGACTCCTTTAACAACAA \\
\hline \multirow[t]{2}{*}{ GAPDH } & F: GCACCGTCAAGGCTGAGAAC \\
\hline & R: TGGTGAAGACGCCAGTGGA \\
\hline
\end{tabular}

F, forward; R, reverse; TNF- $\alpha$, tumor necrosis factor- $\alpha$; IFN- $\gamma$, interferon- $\gamma$; TGF- $\beta$, transforming growth factor- $\beta$; IL, interleukin.

with neutral gum and observed under a fluorescence microscope (Leica Microsystems GmbH) at x100 magnification. Peptidyl-prolyl cis-trans isomerase B (Aviva Systems Biology, San Diego, CA, USA) was used as the internal control.

Candidate IncRNA length sequence and verification of protein coding function. Full-length candidate lncRNAs were established via 5' and $3^{\prime}$ rapid amplification of cDNA ends (RACE) usingaFirstChoice ${ }^{\circledR}$ RLM-RACEkit(Invitrogen; ThermoFisher Scientific, Inc.). Candidate IncRNA non-coding verification was performed by cloning the full-length ENST00000411554 sequence into pcDNA3.1 vectors (Guangzhou RiboBio Co., Ltd.). A total of three Myc-tagged proteins encoding three different sequences were inserted into the $\mathrm{C}$-terminus of the lnc-testicular cell adhesion molecule 1, pseudogene. In addition, the full-length ENST00000411554 sequence was also cloned into pFlag-CMV-2 vectors (Guangzhou RiboBio Co., Ltd.) containing Flag-tagged protein at the $\mathrm{N}$-terminus using three different cloning techniques (16). The plasmids were mixed with Lipofectamine ${ }^{\circledR} 2000$ (Invitrogen; Thermo Fisher Scientific, Inc.) and transferred into 293T cells $\left(1 \times 10^{6} /\right.$ vector, Invitrogen; Thermo Fisher Scientific, Inc.). pcDNA3.1 vectors containing a Myc-tagged green fluorescent protein (GFP) or pFlag-CMV-2 containing a Flag-linked GFP were used as positive controls. An empty plasmid was used as the negative control. The total RNA was isolated and subjected to RT-qPCR to determine the vector-mediated gene transduction 
Table II. Primer sequences for reverse transcription-quantitative polymerase chain reaction of long non-coding (lnc)RNAs.

$\begin{array}{ll}\text { lncRNAs } & \text { Primer sequence }\left(5^{\prime}-3^{\prime}\right)\end{array}$

A, Upregulated

T199454

F: GAGATTGAGAAACTGAGGTCGTG

R: CCCACCACTGATAAGGGATGT

T118424

F: GCCTTCTAAATCACAGACCCC

ENST00000447028

R: AGACTCCCATTTGGAGAGCCTA

ENST00000585911

F: AACACTGGAAACACCCA GCTCTC

R: TCACCACTGCTCAGCCCAGACCTCCCT

ENST00000556606

F: ATCAACTTGTAAGAGAGCTGGGGTTCT

R: AGTTAAACTAGGCCATGATGACAC

TCONS_12_00009699

F: AGAGGTCCCTCAGTGCCAGGGCCT

R: CATGAGACTTATTTTGACT

F: CCACCCAACTCTAAGCATCA

T299457

R: GGTCAAGTAGGCAGTCAGGTAT

F: GCTCCTGTGGGGAATAGACT

NR_029393

R: CCTCACCTCCTCTACTACCAAGA

F: AACACACTGATTCCCATGGCTGAATA

T381695

R: TCCTTGAAGATCACAGGGGTGGT

F: CATGAGACTTATTTTGACTTCTAC

R: GAAGTGGGTGCAGAGGCTGGG

ENST00000566954

F: GCCCTCTTCTTCAAGGATGC

R: GCGGGCACATTTCACAGAT

$\beta$-actin

F: GTGGCCGAGGACTTTGATTG

R: CCTGTAACAACGCATCTCATATT

B, Downregulated

TCONS_00019680

F: ACCACTATGCCTGTGGTGGC

R: ATATCTAGATCTGTGTGC

$\mathrm{T} 173832$

F: CAATGACGCACAGGAGAAAG

ENST00000416861

R: AGATAAGCTTCTTGCCTGT

F: AGCAGGCACCTCTTATGCT

ENST00000527239

R: TTCTCCACATTATTCTCCT

ENST00000561322

F: GGTTGCTGCTCTCCATGAG

R: ATCAGGCAGAGAGAGA

F: TACCGGTACAGCCGGGCTTCAAT

R: CTGGCAGCCAACCACGCAGA

$\mathrm{T} 182081$

F: GCCGACCCCCTGAGGCTCGC

R: CACTCCATCCGGACCAGGG

ENST00000411554

F: ATAAAGTTTTACTTTATACG

T071762

R: TACCAGGAGACATGAGA

F: CTCACAGCAGATCTCTCTGGCTTAAC

R: GTGCTCCAGTTCTTATGGTGTT

ENST00000430816

F: TGCCCAGAAGGCTCTGGAAGA

R: TTCTGGAAGTAAGCACGG

ENST00000606648

F: GTGTTGTAGAATAGGAGGGTCCTGG

R: ATATGAAAACCCAAATGGAGTGAAT

$\beta$-actin

F: GTGGCCGAGGACTTTGATTG

R: CCTGTAACAACGCATCTCATATT

F, forward; R, reverse. 


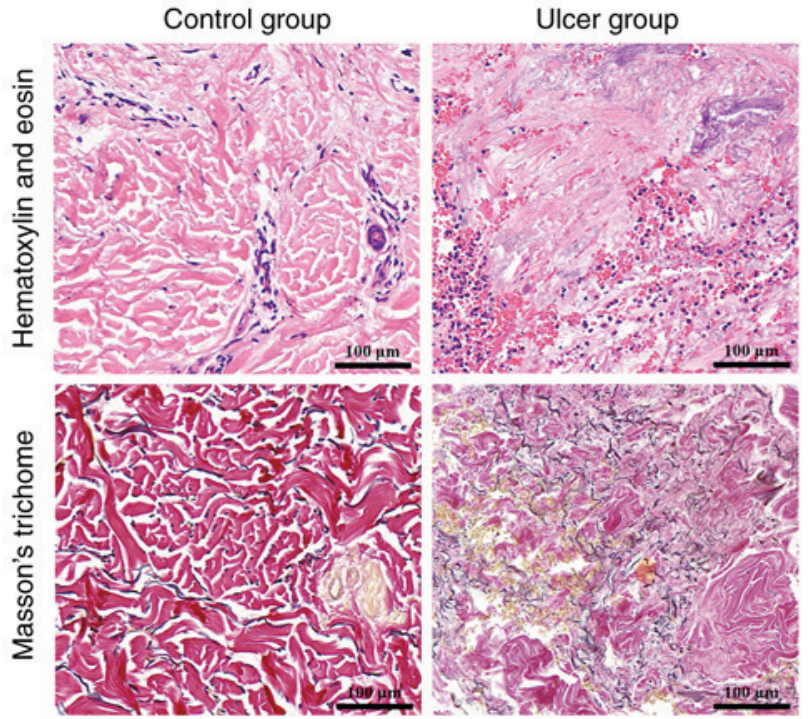

Figure 1. Hematoxylin and eosin and Masson's trichome staining revealing severe morphological structural changes and inflammatory cell infiltration in the ulcer group compared with the control group.

$48 \mathrm{~h}$ post-transfection. After a 72-h time interval, the protein was extracted and Myc-tagged or Flag-tagged proteins were detected via western blotting as described above.

Statistical analysis. All experiments were repeated at least three times. All statistical data are expressed as the mean \pm standard deviation. Statistical analysis was performed using the SPSS 18.0 software package (SPSS, Inc., Chicago, IL, USA). Comparisons of the measurement data between two groups were performed using a Student's t-test. Pearson's test was used for correlation analysis. $\mathrm{P}<0.05$ was considered to indicate a statistically significant difference.

\section{Results}

Histopathological changes at the wound surface of the control and ulcer groups. HE staining of the control wound surface revealed a clear tissue structure with numerous neutrophil granulocytes and fibroblast infiltration, while Masson staining revealed thin collagenous fibers in a loose arrangement. HE staining of the ulcer group indicated a disordered tissue structure, the formation of granulated tissue, increased numbers of neutrophil granulocytes and fibroblast infiltration. Masson staining of the ulcer group revealed atrophic and decreased numbers of collagenous fibers in a disordered arrangement (Fig. 1).

Differential expression of a T lymphocyte subset in the wound surface of the control and ulcer groups. Immunofluorescence staining revealed increased expression of CD3 and CD8 in the wound surface of tissues obtained from the ulcer group compared with the control group; however, there was no marked difference in the levels of CD4 expression between the two groups (Fig. 2). These results indicated a decreased CD4:CD8 ratio in DFU tissues, which suggested that cellular immune dysfunction was present in patients with type $2 \mathrm{DM}$ and DFUs.

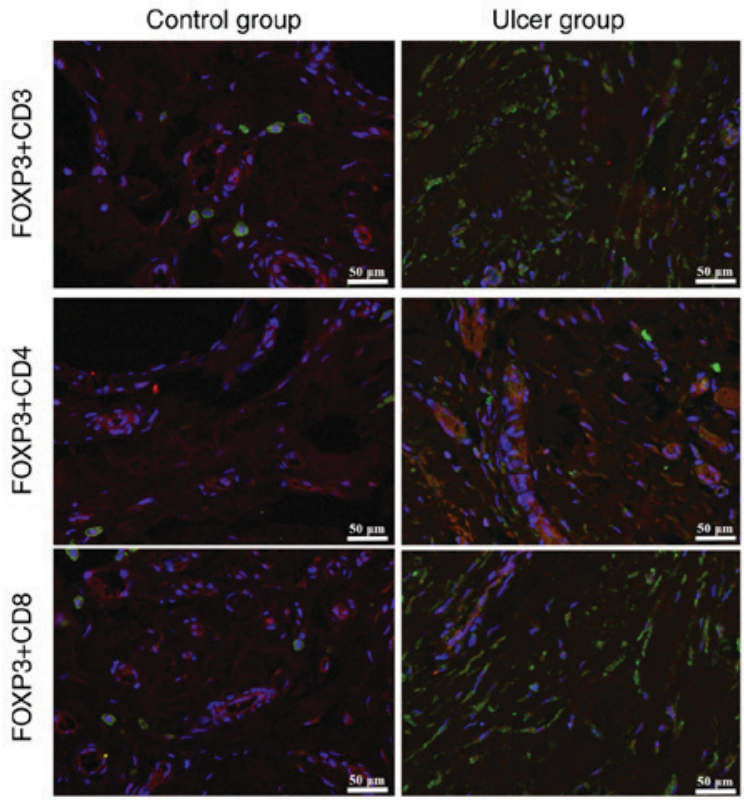

Figure 2. Immunofluorescence staining revealing increased expression levels of CD3 and CD8 in the wound surface of diabetic foot ulcers compared with controls. There was no significant difference in CD4 expression levels between the two groups (green staining indicates CD3, CD4 and CD8 expression; red staining indicates FOXP3 expression; and blue staining indicates DAPI staining). CD, cluster of differentiation; FOXP3, forkhead box P3.

Differential expression of inflammatory factors in the wound surface of the control and ulcer groups. RT-qPCR and western blotting demonstrated that IL-1 $\beta$, IL-2, IL-10, IFN- $\gamma$ and TNF- $\alpha$ expression levels were significantly upregulated in skin tissues obtained from the ulcer group compared with the control group; however, no significant differences in the expression levels of TGF- $\beta$, IL- 6 and IL- 8 were revealed between the two groups (Fig. 3). These results suggested that IL-1 $\beta$, IL-2, IL-10, IFN- $\gamma$ and TNF- $\alpha$ may be associated with the onset of DFUs.

Differential expression of lncRNAs in the wound surface of the control and ulcer groups. To investigate the involvement of lncRNA in DFUs, IncRNA/mRNA gene chip technology was used to detect lncRNA and mRNA expression in the wound surface tissues obtained from the ulcer and control groups. The expression levels of 2,142 lncRNAs were revealed to be upregulated, while 1,332 lncRNAs were downregulated with a differential multiple of $>2$. A total of 20 lncRNAs exhibiting significant differential expression between the two groups were selected for subsequent RT-qPCR analysis. The results demonstrated that five lncRNAs were upregulated, while eight lncRNAs were downregulated, which was consistent with the results of the chip screening analyses (Fig. 4).

Differential expression of IncRNA-ENST00000411554/mitogen activated protein kinase 1 (MAPK1) in control and DFU tissues. Following the analysis of the aforementioned 13 lncRNAs and target genes, it was revealed that IncRNA-ENST00000411554 (location, chromosome 22: 22,390,743-22,394,463; transcript length, $779 \mathrm{bp}$ ) was located in a key upstream regulatory region of target gene, $M A P K 1$. Of the numerous signaling pathways involved in the regulation of DFU, the MAPK 
A

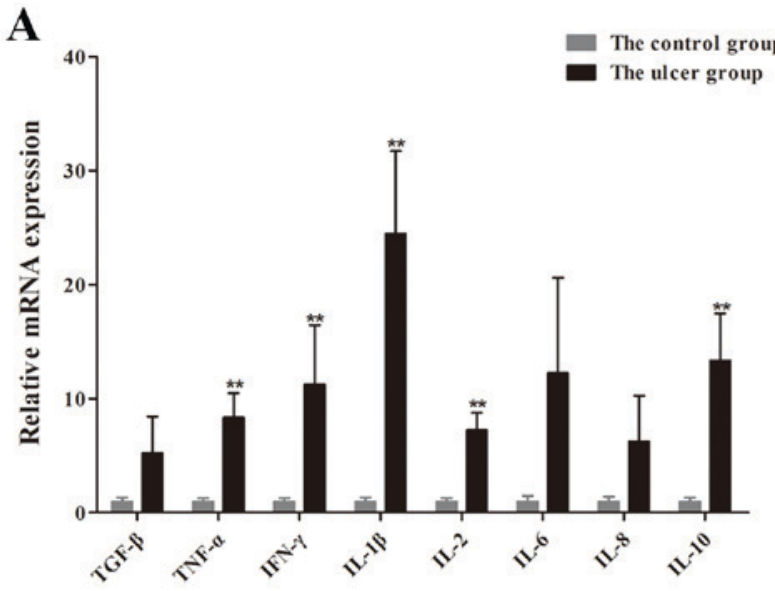

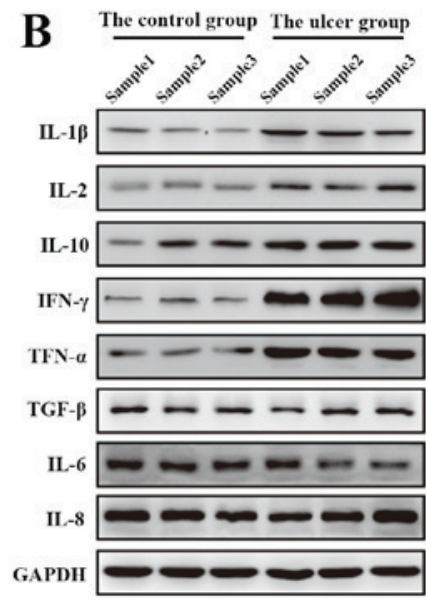

Figure 3. Differential expression of inflammatory factors in wounds of normal and diabetic foot ulcers. (A) Reverse transcription-quantitative polymerase chain reaction and (B) western blotting revealed that the expression levels of IL-1 $\beta$, IL- 2 , IL-10, IFN- $\gamma$ and TNF- $\alpha$ were upregulated in skin tissues obtained from the ulcer group compared with the control group; however, the expression levels of TGF- $\beta$, IL- 6 and IL-8 did not exhibit any significant differences between the two groups. ${ }^{* *} \mathrm{P}<0.01$ vs. respective control. IL, interleukin; IFN- $\gamma$, interferon- $\gamma$; TNF- $\alpha$, tumor necrosis factor- $\alpha$; TGF- $\beta$, transforming growth factor- $\beta$.

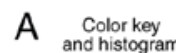

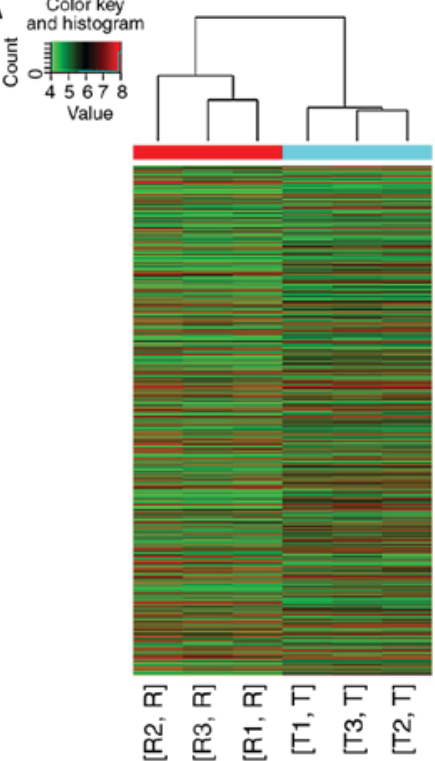

$\mathrm{C}$

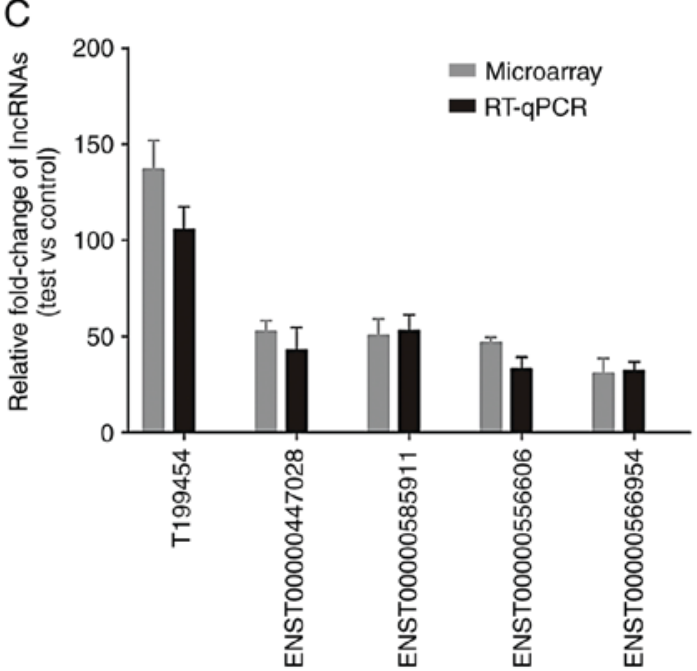

B
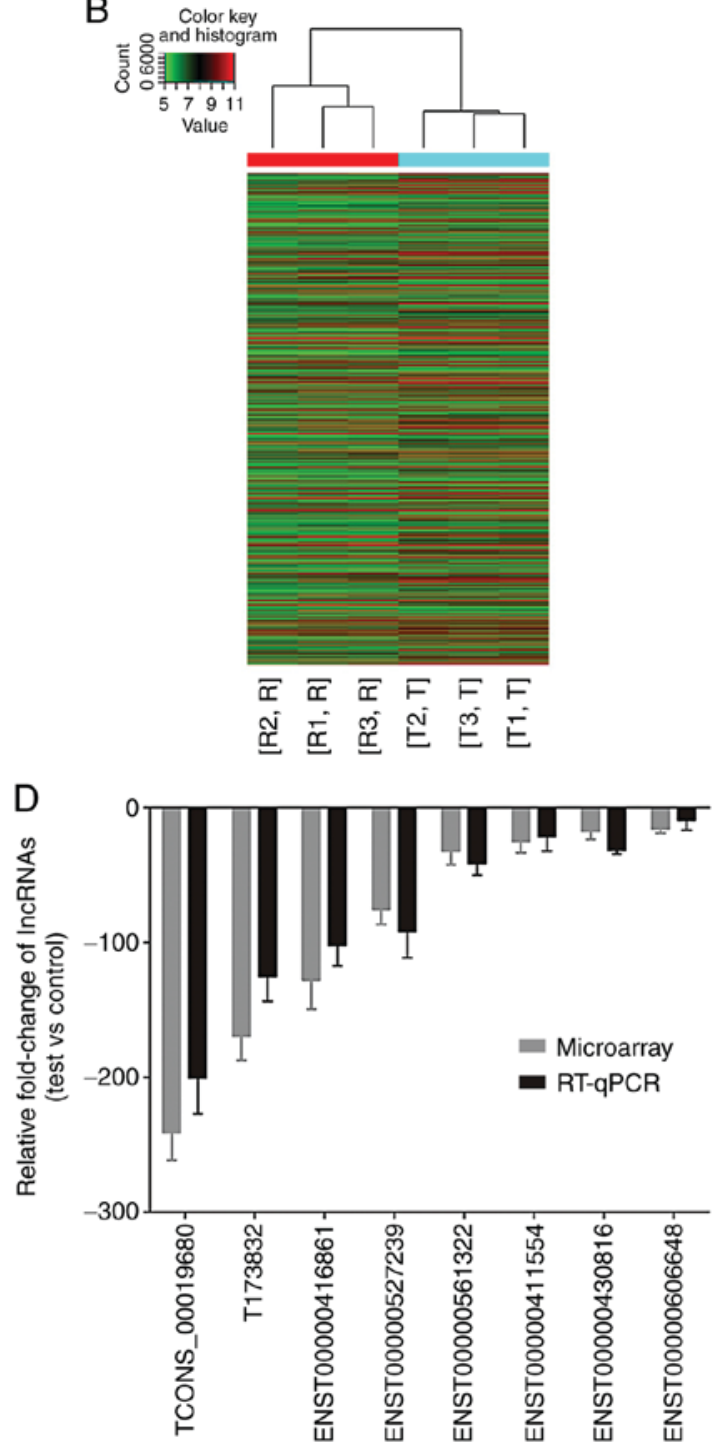

Figure 4. Screening and verification of lncRNAs in the wound surfaces of the control and ulcer groups. (A) lncRNA and (B) mRNA microarray cluster analysis. (C) Verification via RT-qPCR screened out 5 upregulated differentially lncRNAs. (D) Verification via RT-qPCR screened out 8 downregulated differentially lncRNAs. IncRNAs, long non-coding RNAs; RT-qPCR, reverse transcription-quantitative polymerase reaction. 

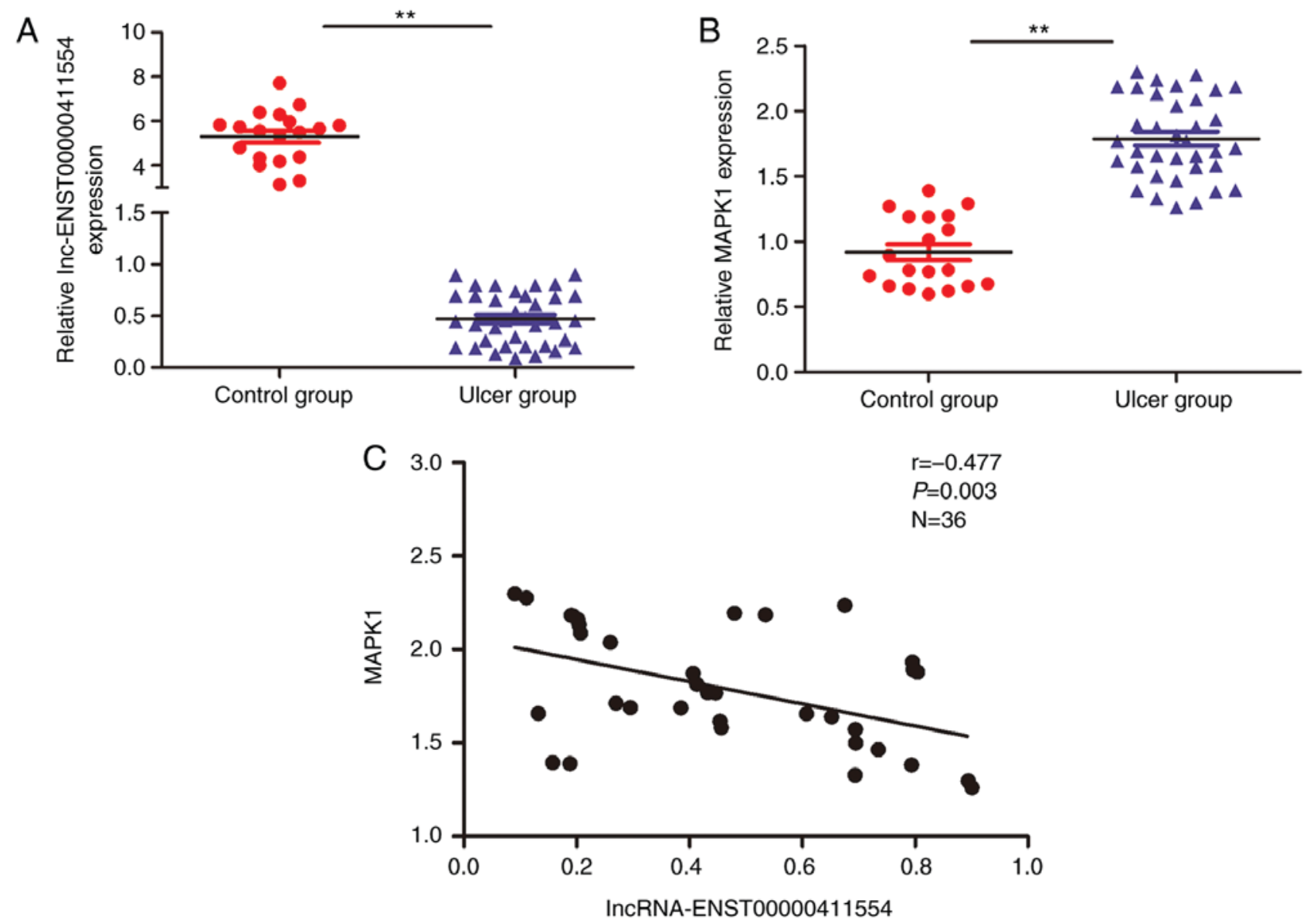

Figure 5. IncRNA-ENST00000411554 is negatively correlated with MAPK1 expression in normal and diabetic foot ulcer tissues. Reverse transcription-quantitative polymerase chain reaction revealed that (A) lncRNA-ENST00000411554 and (B) MAPK1 exhibited differential expression in the control and ulcer groups. ${ }^{* *} \mathrm{P}<0.01$. (C) Expression of IncRNA-ENST00000411554 exhibited a negative correlation with MAPK1 expression in diabetic foot ulcer tissues. MAPK1, mitogen-activated protein kinase 1; lncRNA, long non-coding RNA.

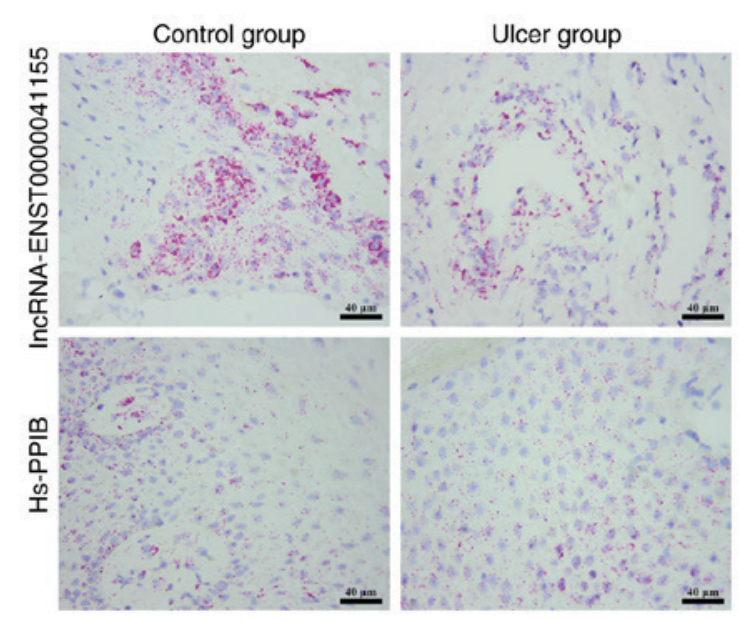

Figure 6. IncRNA fluorescence in situ hybridization demonstrates the differential expression of lncRNA-ENST00000411554 in control and diabetic foot ulcer tissues. Red staining indicates Hs-PPIB or lncRNA-ENST00000411554; blue staining indicates DAPI. lncRNA, long non-coding RNA; Hs-PPIB, Homo sapiens Peptidyl-prolyl cis-trans isomerase B.

signal transduction pathway is one of the most important (17). Therefore, to investigate whether there was a negative correlation between IncRNA-ENST00000411554 and MAPK1 expression in control and DFU tissues, an additional 19 control and 36 ulcer wound surface tissue samples were investigated via lncRNA FISH and RT-qPCR. The results revealed that lncRNA-ENST00000411554 expression was significantly downregulated and $M A P K 1$ expression was significantly upregulated in DFU tissues compared with the control tissues, and thus exhibited a negative correlation (Fig. 5). lncRNA FISH also revealed differential expression of lncRNA-ENST00000411554 in control and DFU tissues (Fig. 6).

lncRNA-ENST00000411554 exhibits non-coding activity. To verify the non-coding characteristics of lncRNA-ENST00000411554, lncRNA-ENST00000411554 was cloned into pcDNA3.1 and pFlag-CMV-2 vectors and subsequently tagged with Myc or Flag, respectively. Following this, these vectors were transfected into $293 \mathrm{~T}$ cells and investigated via western blotting. The results did not reveal any detection of Myc- or Flag-tagged proteins via western blotting, which suggested that IncRNA-ENST00000411554 does not have any protein-coding abilities (Fig. 7).

\section{Discussion}

The sharp increase in DM incidence worldwide has resulted in DFUs becoming an increasingly common complication, with an 

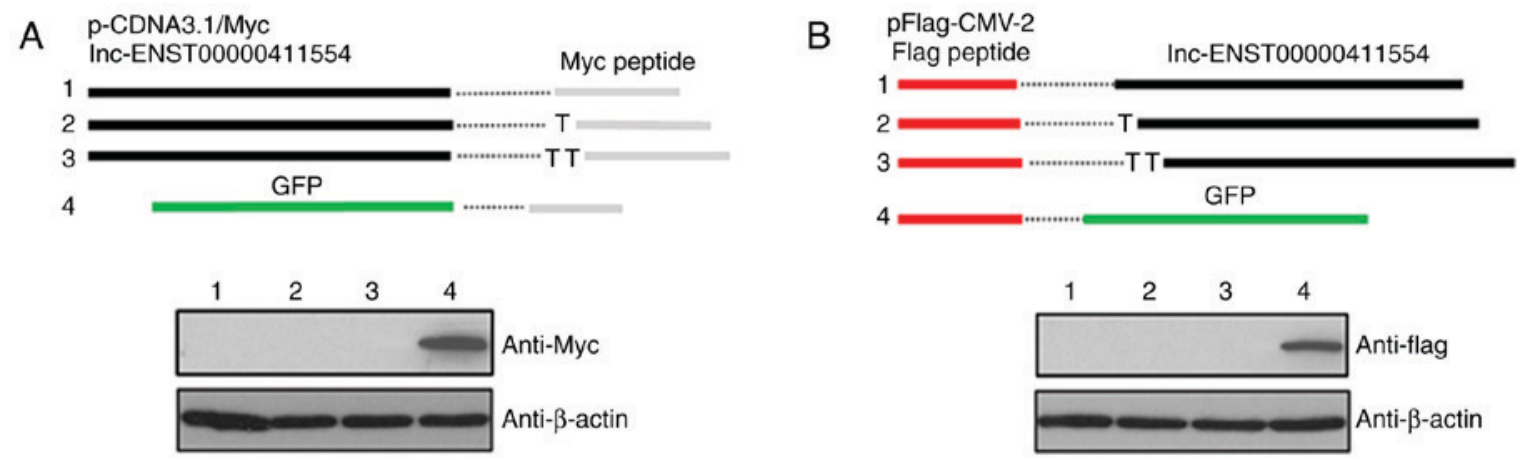

Figure 7. IncRNA-ENST00000411554 non-coding activity verification. (A) 1,2 and 3 indicate lncRNA + the three different encoded modes of Myc-tagged proteins. 4 indicates the GFP protein coding sequence + Myc-tagged protein. Western blot analysis demonstrated that no coding protein was produced, but GFP protein was produced normally. (B) 1,2 and 3 indicate lncRNA + the three different encoded modes of Flag-tagged proteins. 4 indicates the GFP protein coding sequence + Flag-tagged protein. Western blot showed that no coding protein was produced, but GFP protein was produced normally. lncRNA, long non-coding RNA.

estimated development risk of $15-25 \%$ in patients with DM (6). Although efforts have been made to control the deterioration of DF, patients with existing DFUs face possible amputation, and the number of associated mortalities is increasing annually. DM involves the immune system and abnormal inflammatory responses, in addition to aberrant adhesion, uptake, chemotaxis and the germicidal function of neutrophil granulocytes, which have been observed in the peripheral blood of patients with DM $(18,19)$. In addition, reduced immunocompetence is one of the risk factors associated with type $2 \mathrm{DM}$ and diabetic macrovascular and microvascular complications (20). Dysregulated levels of pro-inflammatory cytokines are one of the principal factors associated with poor healing of DFU wound surfaces, and thus represent a key focus of treatment.

Previous studies have indicated that $\mathrm{T}$ lymphocytes, vasculopathy and DFU infection are closely associated in patients with DM (21). The T lymphocyte subset is the most important immune cell population (22). CD3, CD4 and CD8 expression levels are determined to investigate whole $\mathrm{T}$ cells, helper $\mathrm{T}$ lymphocytes and suppressor/cytotoxic T lymphocytes, respectively. The regulatory association between CD4 and CD8 expression has both adjuvant and inhibitory effects, and their ratio is important for immunoregulation (23). Following the stimulation of CD8 cells by extrinsic antigens, cell-mediated killing is induced, which directly destroys target cells. However, following the sensitization of CD4 cells by extrinsic antigens, numerous cytokines are generated to induce the proliferation of $\mathrm{T}$ cells, B cells and macrophages.

In the present study, the results demonstrated that the expression levels of CD3 and CD8 in the ulcer group were increased; whereas levels of CD4 did not exhibit a significant difference between the ulcer group and the control group. Furthermore, the $\mathrm{CD} 4 / \mathrm{CD} 8$ ratio was decreased in patients with $\mathrm{DFU}$, which suggested that patients in the ulcer group suffered from severe immune dysfunction. Despite inflammatory factors having an important role in the immune response, it is unclear which $\mathrm{T}$ lymphocyte subset infiltration has the major effect and which cytokines are involved in inflammation. To investigate this, expression levels of $\mathrm{T}$ lymphocyte-associated inflammatory factors were detected, and the results revealed that IL-2, IFN- $\gamma$ and TNF- $\alpha$ expression levels were significantly upregulated in the ulcer group compared with the control group. Therefore, it may be suggested that following the infection of keratinocytes surrounding the ulcer wound surface, TNF- $\alpha$ is released from inflammatory infiltrating cells and subsequently activates $\mathrm{CD}^{+} \mathrm{T}$ cells and IL-2, which further enhances the secretion of TNF- $\alpha$ and TNF- $\gamma$.

Among the various signaling pathways involved in the regulation of DFUs, the MAPK signal transduction pathway is particularly important (24). The extracellular signal-related kinase (ERK) pathway is also considered to be an important MAPK signaling pathway, and has been revealed to have a crucial role in the inflammatory response induced by $\mathrm{T}$ cells by regulating the expression of inflammatory factors. Kremer et al (25) demonstrated that the ERK pathway activates activator protein-1 (AP-1) in T cells via a phosphorylation cascade, and that p-AP-1 upregulates IL-10 expression by directly binding to its promoter region. IFN- $\gamma$ and IL-1 $1 \beta$ pro-inflammatory factors are also regulated by the ERK pathway $(26,27)$. In the present study, the results revealed that MAPK1, IFN- $\gamma$ and IL- $1 \beta$ were upregulated in DFU tissues, which suggested that MAPK1 promoted the occurrence and development of DFUs. The analysis of lncRNA/mRNA expression profile changes via gene chip technology demonstrated that IncRNA-ENST00000411554 binds to MAPK1. Therefore, it may be suggested that IncRNA-ENST00000411554 induces the upregulation of MAPK1 at the DFU wound surface. Furthermore, the results revealed that lncRNA-ENST0000041155 exhibited non-coding characteristics, and was negatively correlated with MAPK1 expression in DFU tissues.

The present study had certain limitations. Only 6 patients were selected for inclusion in the lncRNA gene chip analysis. Future studies may expand the number of study participants. In addition, there was no corresponding IncRNA knockout and functional verification test to further clarify the function of lncRNA-ENST0000041155. This may be included in subsequent studies.

In conclusion, cellular immune dysfunction is an important factor associated with the occurrence and development of DFUs. Furthermore, the results of the present study suggested that activation of the MAPK signal transduction pathway mediated by lncRNA-ENST00000411554/MAPK1 is associated with an imbalance in DFU immune regulation; however, this requires further investigation. 


\section{Acknowledgements}

Not applicable.

\section{Funding}

The present study was supported by a grant from The National Natural Science Foundation of China (grant no. 81572185), The Natural Science Foundation of Anhui Province of China (grant no. 1708085MH185), The Chinese Anhui province Education Department key Fund Project (grant no. KJ2018A0252) and The Chinese Wuhu City Scientific and Technological Achievements Transformation Project (grant no. 2017CG27).

\section{Availability of data and materials}

The datasets used and analyzed during the current study are available from the corresponding author on reasonable request.

\section{Authors' contributions}

SX conceived the idea of the study. XW, YW, DL, MZ and FZ performed the research. YS analysed data and interpreted the results. SX wrote the paper. All authors discussed the results and revised the manuscript.

\section{Ethics approval and consent to participate}

The present study was approved by the Ethics Committee of Yijishan Hospital (Wuhu, China), and all patients provided written informed consent.

\section{Patient consent for publication}

This study was approved by the Ethics Committee of Yijishan Hospital (Wuhu, China). Written informed consent was obtained from all enrolled patients. All the subsequent research analyses were carried out in accordance with the approved guidelines.

\section{Competing interests}

The authors declare that they have no competing interests.

\section{References}

1. Leung PC: Diabetic foot ulcers-a comprehensive review. Surgeon 5: 219-231, 2007.

2. Unwin N: The diabetic foot in the developing world. Diabetes Metab Res Rev 24 (Suppl 1): S31-S33, 2008.

3. Xu Y, Wang L, He J, Bi Y, Li M, Wang T, Wang L, Jiang Y, Dai M, Lu J, et al: Prevalence and control of diabetes in Chinese adults. JAMA 310: 948-959, 2013.

4. Izumi Y, Satterfield K, Lee S and Harkless LB: Risk of reamputation in diabetic patients stratified by limb and level of amputation: A 10-year observation. Diabetes Care 29: 566-570, 2006.

5. Lepore G, Maglio ML, Cuni C, Dodesini AR, Nosari I, Minetti B and Trevisan R: Poor glucose control in the year before admission as a powerful predictor of amputation in hospitalized patients with diabetic foot ulceration. Diabetes Care 29: 1985, 2006.

6. Boulton AJ: The diabetic foot: A global view. Diabetes Metab Res Rev 16 (Suppl 1): S2-S5, 2000.
7. Zykova SN, Svartberg J, Seljelid R, Iversen H, Lund A, Svistounov DN and Jenssen TG: Release of TNF-alpha from in vitro-stimulated monocytes is negatively associated with serum levels of apolipoprotein B in patients with type 2 diabetes. Scand J Immunol 60: 535-542, 2004.

8. Shau H, Gupta RK and Golub SH: Identification of a natural killer enhancing factor (NKEF) from human erythroid cells. Cell Immunol 147: 1-11, 1993.

9. Thorsby P, Undlien DE, Berg JP, Thorsby E and Birkeland KI: Diabetes mellitus-a complex interaction between heredity and environment. Tidsskr Nor Laegeforen 118: 2519-2524, 1998 (In Norwegian).

10. Bouter KP, Meyling FH, Hoekstra JB, Masurel N, Erkelens DW and Diepersloot RJ: Influence of blood glucose levels on peripheral lymphocytes in patients with diabetes mellitus. Diabetes Res 19: 77-80, 1992.

11. Morán I, Akerman I, van de Bunt M,Xie R, Benazra M, Nammo T, Arnes L, Nakić N, García-Hurtado J, Rodríguez-Seguí S, et al: Human $\beta$ cell transcriptome analysis uncovers lncRNAs that are tissue-specific, dynamically regulated, and abnormally expressed in type 2 diabetes. Cell Metab 16: 435-448, 2012.

12. Gao Y, Wu F, Zhou J, Yan L, Jurczak MJ, Lee HY, Yang L, Mueller M, Zhou XB, Dandolo L, et al: The H19/let-7 double-negative feedback loop contributes to glucose metabolism in muscle cells. Nucleic Acids Res 42: 13799-13811, 2014.

13. Wu G, Cai J, Han Y, Chen J, Huang ZP, Chen C, Cai Y, Huang H, Yang Y, Liu Y, et al: LincRNA-p21 regulates neointima formation, vascular smooth muscle cell proliferation, apoptosis, and atherosclerosis by enhancing p53 activity. Circulation 130: 1452-1465, 2014.

14. Alvarez ML and DiStefano JK: Functional characterization of the plasmacytoma variant translocation 1 gene (PVT1) in diabetic nephropathy. PLoS One 6: e18671, 2011.

15. Livak KJ and Schmittgen TD: Analysis of relative gene expression data using real-time quantitative PCR and the 2(-Delta Delta $\mathrm{C}(\mathrm{T}))$ method. Methods 25: 402-408, 2001.

16. Huang JZ, Chen M, Chen, Gao XC, Zhu S, Huang H, Hu M, Zhu H and Yan GR: A peptide encoded by a putative lncRNA HOXB-AS3 suppresses colon cancer growth. Mol Cell 68: 171-184 e6, 2017.

17. Yang CT, Chen L, Chen WL, Li N, Chen MJ, Li X, Zheng X, Zhao YZ, Wu YX, Xian MA and Liu J: Hydrogen sulfide primes diabetic wound to close through inhibition of NETosis. Mol Cell Endocrinol 480: 74-82, 2019.

18. Lipsky BA, Peters EJ, Berendt AR, Senneville E, Bakker K, Embil JM, Lavery LA, Urbančič-Rovan V and Jeffcoate WJ; International Working Group on Diabetic Foot: Specific guidelines for the treatment of diabetic foot infections 2011. Diabetes Metab Res Rev 28 (Suppl 1): S234-S235, 2012.

19. Boulton AJ: The pathogenesis of diabetic foot problems: An overview. Diabet Med 46 (Suppl 2): S12-S16, 1996.

20. Navarro-González JF and Mora-Fernández C: The role of inflammatory cytokines in diabetic nephropathy. J Am Soc Nephrol 19: 433-442, 2008.

21. Moura J, Rodrigues J, Goncalves M, Amaral C, Lima M and Carvalho E: Impaired T-cell differentiation in diabetic foot ulceration. Cell Mol Immunol 14: 758-769, 2017.

22. Tanaka S, Isoda F, Ishihara Y, Kimura M and Yamakawa T: T lymphopaenia in relation to body mass index and TNF-alpha in human obesity: Adequate weight reduction can be corrective. Clin Endocrinol (Oxf) 54: 347-354, 2001.

23. Keane WF and Lyle PA; Reduction of Endpoints in NIDDM with the Angiotensin II Receptor Antagonist Losartan study: Recent advances in management of type 2 diabetes and nephropathy: Lessons from the RENAAL study. Am J Kidney Dis 41 (3 Suppl 1): S22-S25, 2003.

24. Yuan X, Han L, Fu P, Zeng H, Lv C, Chang W, Runyon RS, Ishii M, Han L, Liu K, et al: Cinnamaldehyde accelerates wound healing by promoting angiogenesis via up-regulation of PI3K and MAPK signaling pathways. Lab Invest 98: 783-798, 2018.

25. Kremer KN, Kumar A and Hedin KE: Haplotype-independent costimulation of IL-10 secretion by SDF-1/CXCL12 proceeds via AP-1 binding to the human IL-10 promoter. J Immunol 178: 1581-1588, 2007.

26. Jin E, Ren M, Liu W, Liang S, Hu Q, Gu Y and Li S: Effect of boron on thymic cytokine expression, hormone secretion, antioxidant functions, cell proliferation, and apoptosis potential via the extracellular Signal-regulated kinases 1 and 2 signaling pathway. J Agric Food Chem 65: 11280-11291, 2017.

27. Wang XL and Sun Q: Photodynamic therapy with 5-aminolevulinic acid suppresses IFN- $\gamma$-induced K17 expression in HaCaT cells via MAPK pathway. Eur Rev Med Pharmacol Sci 21: 4694-4702, 2017. 